\title{
Fabrication of Hyaluronan-Poly(vinylphosphonic acid)-Chitosan Hydrogel for Wound Healing Application
}

\author{
Dang Hoang Phuc, ${ }^{1}$ Nguyen Thi Hiep, ${ }^{1}$ Do Ngoc Phuc Chau, ${ }^{2}$ Nguyen Thi Thu Hoai, \\ Huynh Chan Khon, ${ }^{1}$ Vo Van Toi, ${ }^{1}$ Nguyen Dai Hai, ${ }^{3}$ and Bui Chi Bao ${ }^{4}$ \\ ${ }^{1}$ Department of Biomedical Engineering, International University, Vietnam National University-Ho Chi Minh City (VNU-HCM), \\ Quarter 6, Linh Trung Ward, Thu Duc District, Ho Chi Minh City 70000, Vietnam \\ ${ }^{2}$ School of Biotechnology, International University, Vietnam National University-Ho Chi Minh City (VNU-HCM), Quarter 6, \\ Linh Trung Ward, Thu Duc District, Ho Chi Minh City 70000, Vietnam \\ ${ }^{3}$ Institute of Applied Materials Science, Vietnam Academy of Science and Technology, Ho Chi Minh City 70000, Vietnam \\ ${ }^{4}$ The Center for Molecular Biomedicine, University of Medicine and Pharmacy, Ho Chi Minh City 70000, Vietnam \\ Correspondence should be addressed to Nguyen Thi Hiep; nthiep1981@gmail.com
}

Received 7 January 2016; Accepted 10 March 2016

Academic Editor: Matthew Green

Copyright (C) 2016 Dang Hoang Phuc et al. This is an open access article distributed under the Creative Commons Attribution License, which permits unrestricted use, distribution, and reproduction in any medium, provided the original work is properly cited.

\begin{abstract}
A new hydrogel made of hyaluronan, poly(vinylphosphonic acid), and chitosan (HA/PVPA/CS hydrogel) was fabricated and characterized to be used for skin wound healing application. Firstly, the component ratio of hydrogel was studied to optimize the reaction effectiveness. Next, its microstructure was observed by light microscope. The chemical interaction in hydrogel was evaluated by nuclear magnetic resonance spectroscopy and Fourier transform-infrared spectroscopy. Then, a study on its degradation rate was performed. After that, antibacterial activity of the hydrogel was examined by agar diffusion method. Finally, in vivo study was performed to evaluate hydrogel's biocompatibility. The results showed that the optimized hydrogel had a threedimensional highly porous structure with the pore size ranging from about $25 \mu \mathrm{m}$ to less than $125 \mu \mathrm{m}$. Besides, with a degradation time of two weeks, it could give enough time for the formation of extracellular matrix framework during remodeling stages. Furthermore, the antibacterial test showed that hydrogel has antimicrobial activity against E. coli. Finally, in vivo study indicated that the hydrogel was not rejected by the immune system and could enhance wound healing process. Overall, HA/PVPA/CS hydrogel was successfully fabricated and results implied its potential for wound healing applications.
\end{abstract}

\section{Introduction}

Bioadhesives, polymers, or copolymers used to join the biological surfaces are frequently used alone or combined with other methods for wound closure applications $[1,2]$. The adhesion property of them is a result of strong bonds, such as covalent, ionic, or metallic bonds, and weak bonds, such as polar, hydrogen, or Van der Waals bonds, between the components [2]. Compared with the conventional subcuticular suture, the bioadhesive showed similar effects on wound healing yet with shorter time, especially when making more than one incision in one patient, and no need of postsurgery revisiting as well as increasing the pain relief $[3,4]$. Studies on facial laceration treatment and repair of laceration in children also proved a significant decrement in time treatment and patients' pain when using a bioadhesive as compared to suture method $[4,5]$. These results suggested that bioadhesives can be a trustworthy substitute for the conventional method. To fabricate bioadhesives, synthetic polymers, natural polymers, or their combination can be used, yet they have to interact with each other as mentioned above and fulfill the requirements: sufficient biodegradation time for wound healing process, high porosity structure to support cell migration, biocompatibility to enhance cell proliferation, producing no toxicity, minimizing inflammation as well as immune response, and lastly antibacterial activity to prevent wounds infections [6]. 
Among the natural polymers, chitosan is one of the most commonly used polymers thanks to its properties of nontoxicity, biodegradability, and biocompatibility as well as its abilities to provide hemostasis and bacteriostasis. Chitosan can be prepared for tissue regeneration applications in various forms, such as gel and membrane [7-10].

Another natural polymer successfully used in wound healing as wound dressing method is hyaluronan [11]. With the properties of noncytotoxicity, nonantigenicity, nonimmunogenicity, and biocompatibility and the abilities to promote cell proliferation $[8,9]$ and induce angiogenesis after being partially degraded $[12,13]$, hyaluronan has also been used in tissue engineering as scaffold, electrospun fiber, solution, and hydrogel $[14,15]$.

On the other hand, in the synthetic group, with a nontoxic property [16, 17], poly(vinylphosphonic acid) (PVPA) has been widely used in tissue engineering in producing dental cements, hydrogels, and scaffolds [18, 19]. Thanks to the abilities to form a three-dimensional (3D) network in hydrogels and interact with serum proteins, PVPA-containing hydrogels have strong mechanical strength and their surface can enhance cell seeding and proliferation [18].

Each polymer has its own advantages; however, they are usually combined to synthesize new materials with specified characteristics for specific applied areas. Fan et al. modified chitosan and hyaluronan with oxanorbornadiene (OB) and 11-azido-3,6,9-trioxaundecan-1-amine (AA), respectively, to fabricate chitosan-hyaluronan hydrogel for soft tissue regeneration [20]. The in vitro and in vivo studies proved that the hydrogel could support the proliferation of human adipose tissue and has a cytocompatible property; hence, it could have a high potential to be used for soft tissue engineering. Chang et al. modified hyaluronan with aldehyded 1-amino3,3-diethoxy-propane (AHA) and fabricated it with chitosan to form the gel AHA-chitosan (AHA-CA) [21]. The results showed that AHA-CA hydrogel could accelerate wound closure of the full-thickness skin defects $(1 \times 1 \mathrm{~cm})$ and enhance keratinocyte migration and cell proliferation, as well as inducing granulation tissue and capillary formation. Besides chitosan and hyaluronan, PVPA was also studied for skin regeneration application. Tan et al. fabricated poly(VPAco-acrylamide) hydrogel and evaluated its protein and cell interaction [18]. The outcomes proved that, by modifying with VPA, the copolymer hydrogel could increase the protein uptake up to two times as compared to polyacrylamide only as well as inducing NIH $3 \mathrm{~T} 3$ fibroblast adhesion and proliferation. Though chitosan, hyaluronan, and PVPA have been studied for skin regeneration and each of them also has its own advantages, the combination of all of them has not been performed elsewhere.

Hence, the aim of this research is to place a first step in combining hyaluronan, chitosan, and PVPA to fabricate a novel HA/PVPA/CS hydrogel to be used as a skin bioadhesive.

In HA/PVPA/CS hydrogel, chitosan could give the hydrogel the hemostasis and bacteriostasis abilities to stop the bleeding and prevent wounds infection. It was hypothesized to form an electrostatic matrix with PVPA to make a highly spacious $3 \mathrm{D}$ porous structure, which could strengthen the mechanical property of the hydrogel and enhance cell seeding [22]. Besides chitosan and PVPA, the addition of hyaluronan to the hydrogel could form the electrolyte complex with chitosan [23], resulting in the increase of the viscosity of the hydrogel, the promotion of cell proliferation, and the induction of angiogenesis. As the primary study for HA/PVPA/CS hydrogel, this research only assessed the essential requirements of the bioadhesive, including (1) highly porous 3D structure to support skin cell migration, seeding, and blood vessel formation; (2) short degradation rate to be suitable for the wound healing process; (3) antibacterial property to prevent infections; and (4) good biocompatibility to enhance cells proliferation as well as not to be rejected by the immune system.

\section{Materials and Methods}

2.1. Materials. Hyaluronan (hyaluronic acid sodium salt from Streptococcus equi), PVPA (poly(vinylphosphonic acid)), and chitosan (chitosan from shrimp shells) were purchased from Sigma Aldrich, USA. Müller-Hinton medium agar was purchased from HiMedia, India. Ciprofloxacin antibiotic discs were purchased from Nam Khoa Co., Ltd., Vietnam. Povidone solution (povidone-iodine 10\%) was purchased from Mekophar Chemical Pharmaceutical Joint-Stock Company, Vietnam. All other chemicals used in this study were purchased from major suppliers, otherwise unmentioned. The materials were used directly without further purification. $E$. coli (Escherichia coli, ATCC35218) suspension with $\mathrm{OD}_{620 \mathrm{~nm}}$ value of $0.08-0.11$ was used for antibacterial experiments. Swiss-albino mice (16 to $20 \mathrm{~g}$ ) were obtained from Pasteur Institute of Ho Chi Minh City, Vietnam, and fed 1 week prior to implantation.

\subsection{Methods}

2.2.1. Optimization of Component Ratio for Hydrogel Preparation. To optimize component ratio of HA/PVPA/CS hydrogel for increasing the reaction effectiveness, the concentration of each component in the hydrogel was evaluated. The ratio was studied step by step, starting with PVPA/CS ratio. To optimize the ratio of PVPA and chitosan in the hydrogel, the concentration of chitosan was studied first, and then the amount of PVPA was evaluated. Firstly, a fixed amount of $200 \mu \mathrm{L}$ PVPA $2 \% \mathrm{w} / \mathrm{v}$ was used and chitosan $1 \% \mathrm{w} / \mathrm{v}$ and chitosan $2 \% \mathrm{w} / \mathrm{v}$ were tested with the amount of $100 \mu \mathrm{L}$ each to obtain the effect of chitosan's concentration on gelation process. After that, the amount and concentration of chitosan were fixed to study the effects of varying the amount of PVPA on the hydrogel. PVPA $10 \% \mathrm{w} / \mathrm{v}$ was added with amounts varying from 20 to $5 \mu \mathrm{L}$ with $5 \mu \mathrm{L}$ decrement steps and the samples were labeled from PCS1 to PCS4 (Table 1). Finally, $\mathrm{HA} / \mathrm{PVPA} / \mathrm{CS}$ ratios were studied based on the PVPA/CS ratio of sample PCS4. The samples were made by using a fixed amount of PVPA and chitosan (following the optimized ratio) and varying the amount of hyaluronan $1 \% \mathrm{w} / \mathrm{v}$ added from 100 to $12.5 \mu \mathrm{L}$ and labeled from HPCS1 to HPCS4 (Table 2). All samples used for in vitro antibacterial activity 
TABLE 1: Amounts of components in PVPA/CS hydrogels.

\begin{tabular}{lcccc}
\hline \multirow{2}{*}{ Components } & \multicolumn{4}{c}{ Samples } \\
& PCS1 & PCS2 & PCS3 & PCS4 \\
\hline PVPA & $20 \mu \mathrm{L}$ & $15 \mu \mathrm{L}$ & $10 \mu \mathrm{L}$ & $5 \mu \mathrm{L}$ \\
CS & $100 \mu \mathrm{L}$ & $100 \mu \mathrm{L}$ & $100 \mu \mathrm{L}$ & $100 \mu \mathrm{L}$ \\
\hline
\end{tabular}

TABLE 2: Amounts of components in HA/PVPA/CS hydrogels.

\begin{tabular}{lcccc}
\hline \multirow{2}{*}{ Components } & \multicolumn{4}{c}{ Samples } \\
& HPCS1 & HPCS2 & HPCS3 & HPCS4 \\
\hline HA & $100 \mu \mathrm{L}$ & $50 \mu \mathrm{L}$ & $25 \mu \mathrm{L}$ & $12.5 \mu \mathrm{L}$ \\
PVPA & $5 \mu \mathrm{L}$ & $5 \mu \mathrm{L}$ & $5 \mu \mathrm{L}$ & $5 \mu \mathrm{L}$ \\
CS & $100 \mu \mathrm{L}$ & $100 \mu \mathrm{L}$ & $100 \mu \mathrm{L}$ & $100 \mu \mathrm{L}$ \\
\hline
\end{tabular}

and in vivo implantation experiments were sterilized using UV irradiation at room temperature for 45 minutes.

2.2.2. Hydrogel Characterization. The microstructure of lyophilized sample HPCS4 was observed using a Nikon Eclipse Ti-U inverted microscope (Nikon, Japan). ${ }^{1} \mathrm{H}$ NMR spectrum of sample HPCS4 was obtained by measuring the freeze-dried sample in $\mathrm{D}_{2} \mathrm{O}$ solvent using a Bruker Advance III Ultra Shield Plus $500 \mathrm{MHz}$ spectrometer (Bruker, USA). FT-IR spectra of the hydrogel and its components (hyaluronan, PVPA, and chitosan) were measured with wave number from 4000 to $400 \mathrm{~cm}^{-1}$ using a Tensor 27 FT-IR spectrometer (Bruker, USA).

Degradation property of sample HPCS4 was studied by gravity method. Briefly, the samples were weighed and then immersed in PBS buffer at $37^{\circ} \mathrm{C}$. At different time points, the samples were taken out, dried, and weighed. The percentages of the remaining weights at different time points were recorded in 15 days following (1), where $w d$ is the percentage of weight remaining at day $t$ and $w d(0)$ and $w d(t)$ are initial weight and weight at day $t$, respectively:

$$
w d(\%)=\frac{w d(t)}{w d(0)} \times 100 \% .
$$

Antibacterial activity of HA/PVPA/CS hydrogel and its components was evaluated using agar diffusion method. Briefly, $100 \mu \mathrm{L}$ of E. coli suspension was added and spread evenly on Müller-Hinton agar surface by using sterile glass spreader. Then, $10 \mu \mathrm{L}$ of each sample was dropped on the suspension layer. Ciprofloxacin antibiotic was used as a positive control. The dishes were incubated overnight at $37^{\circ} \mathrm{C}$.

Biocompatibility of hydrogel was evaluated by using mice model. The operation process was performed following the policy of Institutional Animal Care and Use Committee of International University, Vietnam National University-Ho Chi Minh City, Vietnam. Firstly, mice were anesthetized with dimethyl ether, their hair was shaved at their back, and they were fixed on a table. The implanted site was cleaned by povidone solution and PBS buffer and a hole with a diameter of $1 \mathrm{~cm}$ was artificially created. Sample HPCS4 was spread evenly on the wound surface. The implantation was replicated on 5 mice and 3 other mice were used as the control. The wound morphology during the healing process was captured for monitoring. At day 14, samples were extracted. Mice were euthanized using cervical dislocation method. Then, mice's hair was shaved and the samples were extracted. The samples were fixed with formaldehyde $4 \% \mathrm{w} / \mathrm{v}$, stained with Hematoxylin and Eosin (H\&E), and processed to microscopic observation.

2.2.3. Statistical Analysis. All experiments in this research were replicated at least three times. Statistical values were calculated from raw data using Microsoft Excel 2013. Graphs were drawn using SigmaPlot 12 software. The comparison between two sets of measurements was performed using twotailed $t$-test.

\section{Results}

3.1. Optimization of Component Ratio for Hydrogel Preparation. In the first step to optimize HA/PVPA/CS ratio, the effect of concentration of chitosan on hydrogel formation was studied. The results demonstrated that using chitosan $2 \% \mathrm{w} / \mathrm{v}$ gave a gelation time of $12 \pm 1 \mathrm{~s}$, which was faster as compared to that of using chitosan $1 \% \mathrm{w} / \mathrm{v}(15 \pm 1 \mathrm{~s}, p<0.05, n=3)$. Hence, chitosan $2 \%$ was used for the next steps.

In the second step, study on the effect of PVPA concentration on PVPA/CS gelation illustrated that the decrement of PVPA amount in the hydrogels affected the gelation and molecular interaction. As the amount of PVPA decreased from $20 \mu \mathrm{L}$ to $5 \mu \mathrm{L}$, the formation of fibers increased. Sample PCS4 (PVPA/CS ratio was 5/100) (Figure 1(d)), which had the least amount of PVPA, showed the largest amount of fibers and the fastest rate of shrinking after being stretched as compared to other samples. Hence, PCS4 was chosen as the optimum for the last step.

In the final step, the ratio between hyaluronan, PVPA, and chitosan was studied. The results indicated that changing in amount of hyaluronan did not affect the fiber formation yet it affected the molecular interaction. After being stretched, sample HPCS4 (HA/PVPA/CS ratio was $12.5 / 5 / 100$ ), with the least amount of hyaluronan, was the least fragmented (Figure 1(h)) among other samples. Therefore, HPCS4 hydrogel had the optimized component ratio, of which the final concentrations of hyaluronan, PVPA, and chitosan were $0.1 \%$, $0.4 \%$, and $1.7 \%$, respectively.

3.2. Hydrogel Characterization. The microstructure of the hydrogel under light microscopic observation is presented in Figure 2. The sample showed a 3D network with highly porous structure. The pore shape and size of the hydrogel were nonuniform and ranging from about $27 \times 43 \mu \mathrm{m}$ to about $67 \times 127 \mu \mathrm{m}$.

Next, chemical interactions of the components of hydrogels were studied by NMR and FT-IR spectroscopies. ${ }^{1} \mathrm{H}$ NMR spectrum of the hydrogel (Figure 3) presented peak at $1.935 \mathrm{ppm}$, which indicated the presence of $\mathrm{N}$-acetyl group in $\mathrm{N}$-acetyl-D-glucosamine of hyaluronan $[24,25]$. Since there was a small amount of PVPA in the sample, the methine proton of PVPA was presented as a peak at $2.1 \mathrm{ppm}$ [26]. The peaks of nonanomeric protons $\mathrm{H}$ molecules in sugar ring of 


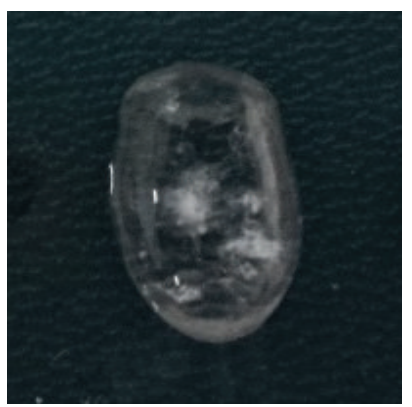

(a)

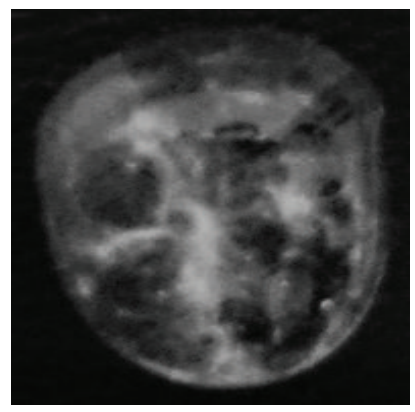

(e)

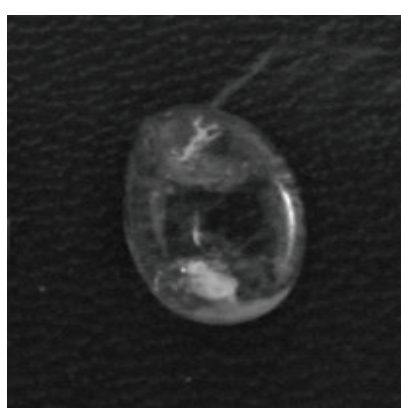

(b)

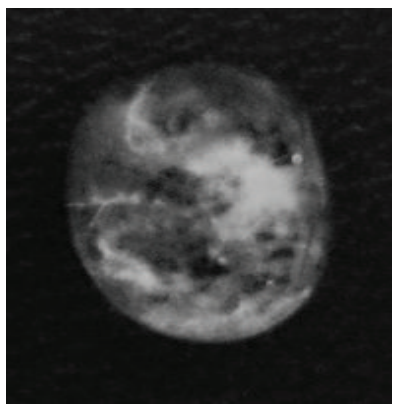

(f)

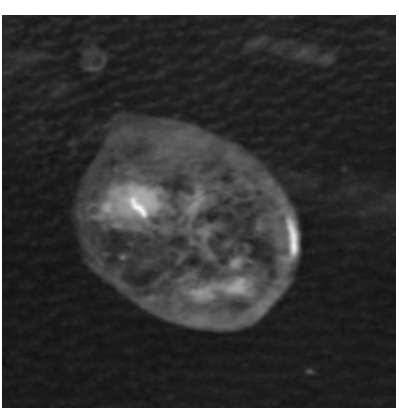

(c)

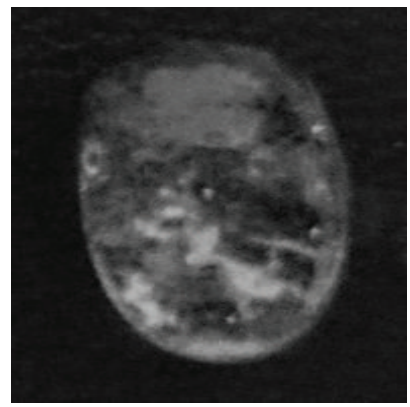

(g)

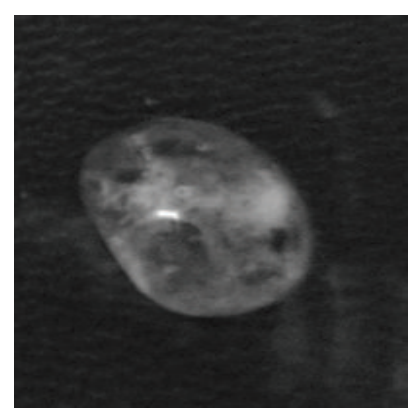

(d)

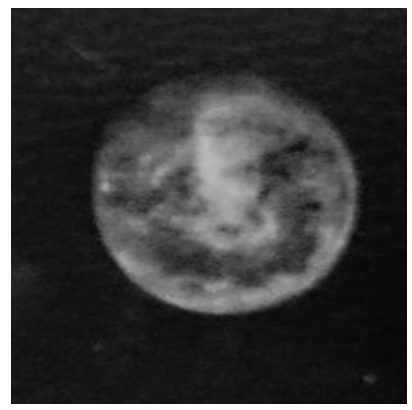

(h)

Figure 1: Morphology of samples PCS1 (a), PCS2 (b), PCS3 (c), PCS4 (d), HPCS1 (e), HPCS2 (f), HPCS3 (g), and HPCS4 (h).

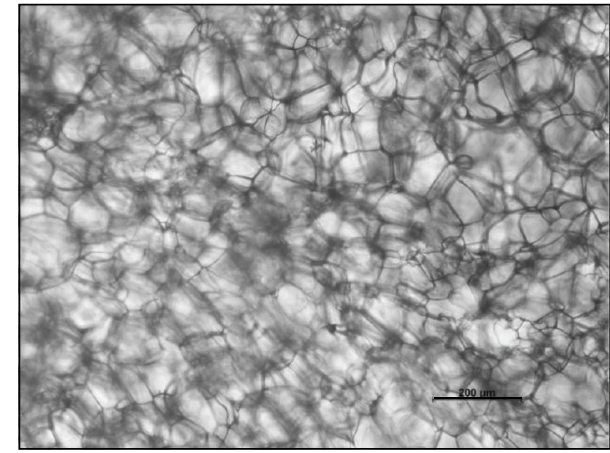

(a)

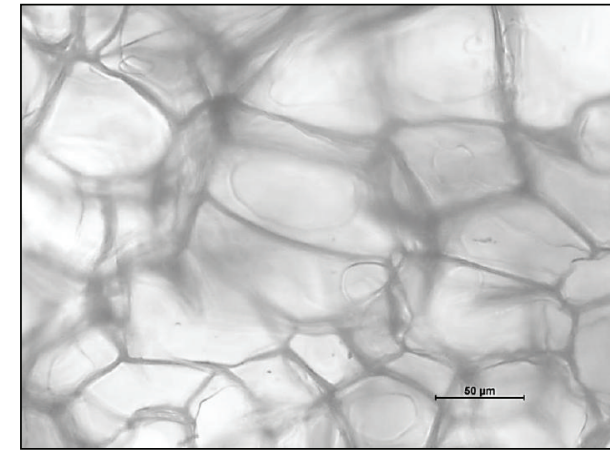

(b)

FIGURE 2: Morphology of sample HPCS4 after being freeze-dried; the pictures were observed with the magnification of 10x and 40x.

hyaluronan and chitosan were overlapped from $3.557 \mathrm{ppm}$ to $3.821 \mathrm{ppm}$. Peak at $4.790 \mathrm{ppm}$ indicated the presence of anomeric proton $\mathrm{H}-1$ molecule in D-glucosamine of chitosan [27]. These results confirmed the presence of HA, PVPA, and CS in its structure.

FT-IR spectra of HPCS4 and its components are presented in Figure 4. The hydrogel shared with CS, HA, and PVPA a peak at $3450 \mathrm{~cm}^{-1}$, which represented $-\mathrm{OH}$ stretching vibration. It also shared the $-\mathrm{C}=\mathrm{O}$ vibration with CS and HA, which was represented by a peak at $1640 \mathrm{~cm}^{-1}$. Besides, peaks at $1414 \mathrm{~cm}^{-1}$, which represented $\mathrm{NH}_{3}{ }^{+}$vibration, confirmed the interaction between the acid groups (carboxyl group and phosphonate group) and base group (primary amine in D-glucosamine) [23, 25, 28, 29].

Degradation assay was studied using PBS buffer at $37^{\circ} \mathrm{C}$ in a period of 15 days and the method of measuring and calculating was described previously in Materials and Methods. Figure 5 showed that the remaining weights after 1,3 , and 6 days were $64 \pm 12 \%, 43 \pm 10 \%$, and $20 \pm 3 \%$, respectively, for the first week. From day 6 to day 15, the weight reduced, yet it was not statistically different. From those results, it can be concluded that HPCS4 had a short degradation time.

The inhibition zones of the hydrogel and its components against $E$. coli are shown in Figure 6. The result revealed that all the materials and samples had antibacterial activity. CS had the clearest inhibition zone with diameter of $0.99 \mathrm{~cm}$ (Figure 6(d)). Inhibition zone of PVPA was observed with diameter of $1.25 \mathrm{~cm}$ (Figure 6(c)). The blurry inhibition zone of HA, with diameter of $1.28 \mathrm{~cm}$, indicated that it had weak antibacterial property (Figure 6(b)). HPCS4 (Figure 6(a)) showed a slightly larger inhibition zone than those of its components $(1.73 \mathrm{~cm})$ and had a medium antibacterial 


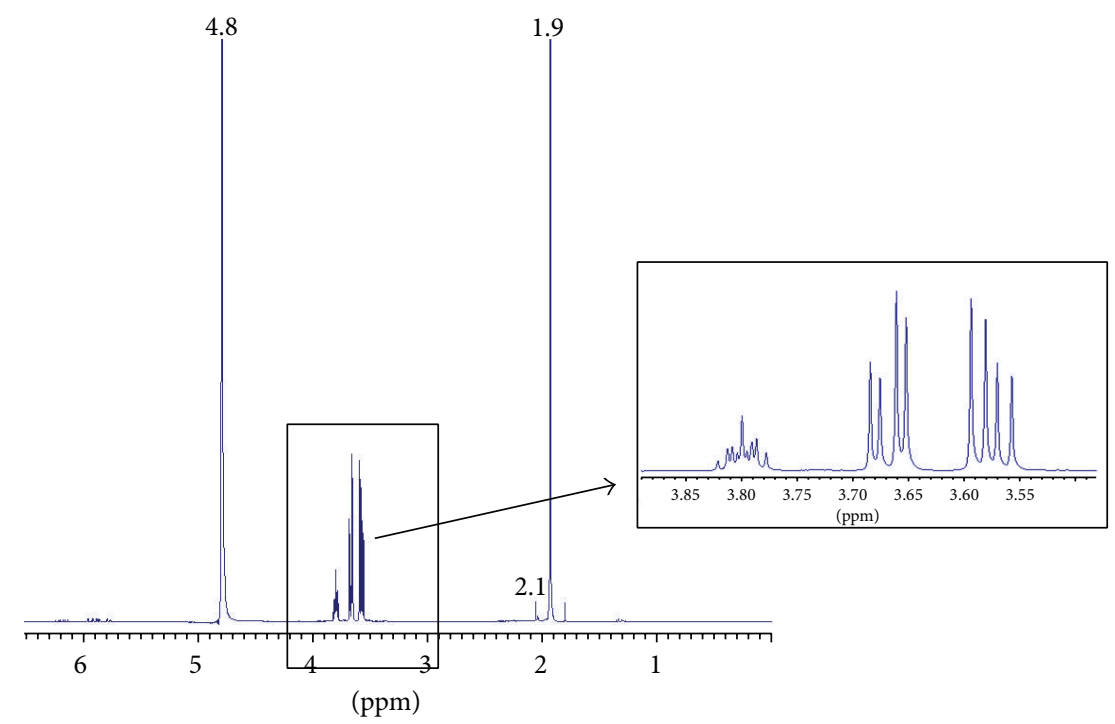

FIGURE $3:{ }^{1} \mathrm{H}$ NMR spectrum of sample HPCS4 and its enlargement (box) from 3.5 to $3.8 \mathrm{ppm}$.

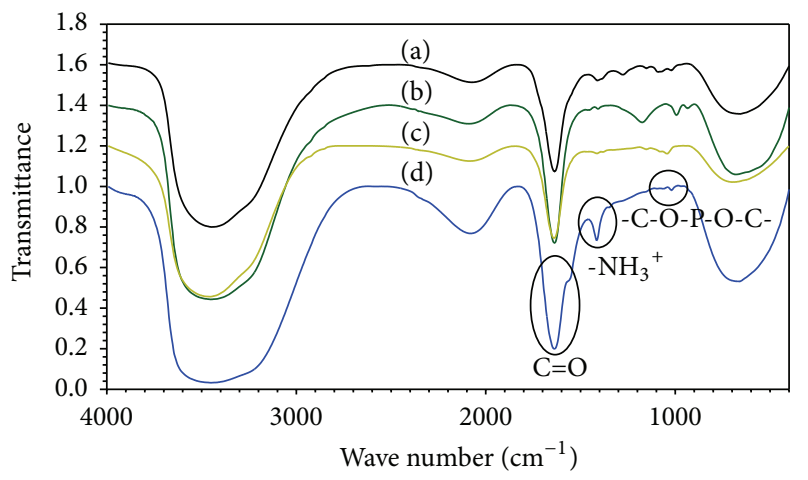

FIGURE 4: FT-IR spectra of CS (a), PVPA (b), HA (c), and HPCS4 (d).

activity as compared to the control Ciprofloxacin antibiotic (Figure 6(e), $2.48 \mathrm{~cm}$ ). From these results, it can be concluded that the hydrogels got antibacterial activity that inhibited $E$. coli proliferation.

The hydrogel's biocompatibility was studied by in vivo murine implantation in duration of 14 days. The wound morphology of the implanted zones and their sizes are shown in Figure 7. Photograph images show that the postimplantation area with the treated hydrogels indicated no sign of inflammation along the period of implantation. The wounds were healed gradually in the first week; their remaining size was $36 \pm 3 \%$ and smaller than that of the control (54 $\pm 8 \%$, $p<0.01, n=5$ ). In the second week, from day 7 to day 10 , wound size rapidly reduced from $36 \pm 3 \%$ to $6.8 \pm 3 \%$ $(p<0.01, n=5)$ and then $2.0 \pm 1 \%$ at day 14 . Comparing the results after 14 days, sample HPCS4 showed that a smaller wound site remained $(2.0 \pm 1 \%)$ as compared to the control $(5.6 \pm 2 \%, p<0.01, n=5)$.

H\&E staining extracted skins of the implanted sites (Figure 8) also indicated aligned results with the morphology. Both opened wounds were closed with new tissue formation,

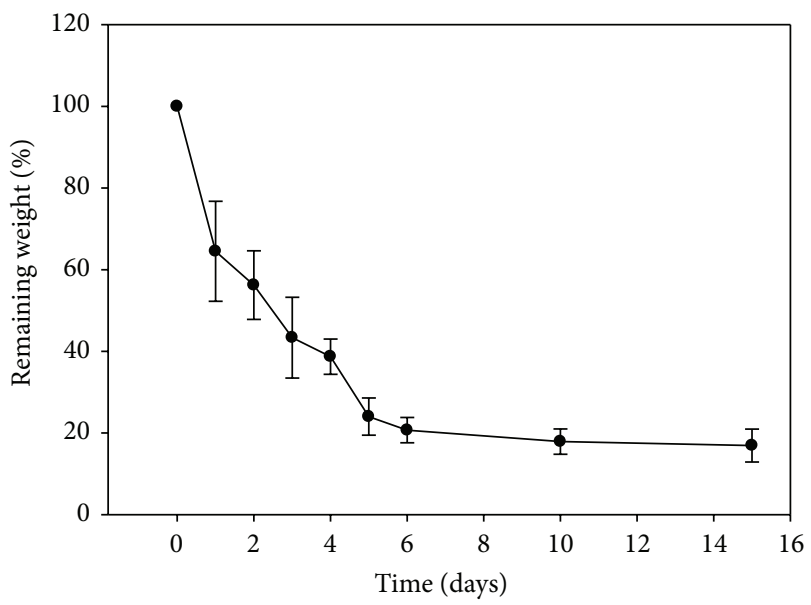

FIgURE 5: Biodegradable test of sample HPCS4 after 16 days in PBS buffer.

arrangement of cells, and formation of capillary. However, new tissue formed differently between the untreated and the treated sample. For example, the untreated wound showed that the wound site was closed but the epidermal tissue layer was not yet formed. In contrast, the treated wound showed that the wound site closed with epidermal layer (Figure 8(d)).

\section{Discussion}

Wounds, without closure treatments, are healed by secondary intention, which may take longer time and leave a scar at the wound sites. Compared to the conventional suture method, the bioadhesive can be considered to have more advantages as it can shorten the treating time and reduce patients' pain during that process. In this research, a combination of hyaluronan, PVPA, and chitosan was used to take advantage of each material in order to fabricate a new hydrogel that 


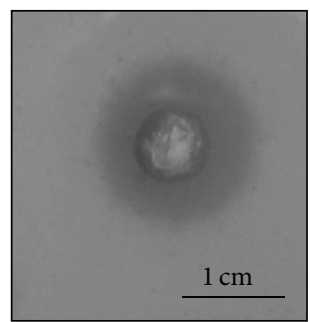

(a)

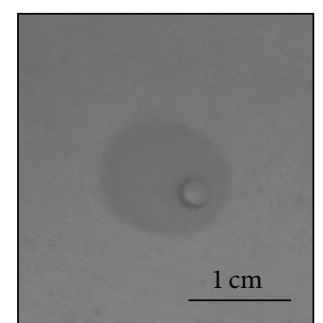

(b)

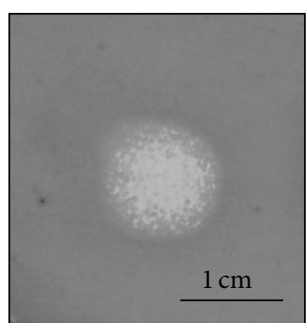

(c)

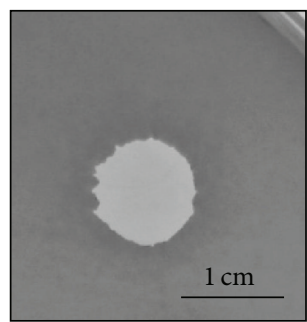

(d)

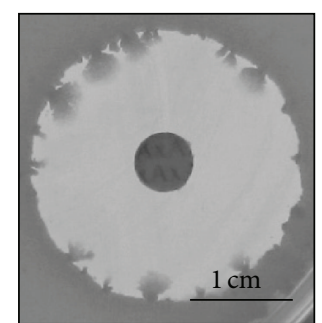

(e)

FIgURE 6: Comparison of antimicrobial activity of samples HPCS4 (a), HA (b), PVPA (c), CS (d), and Ciprofloxacin (e) against E. coli.
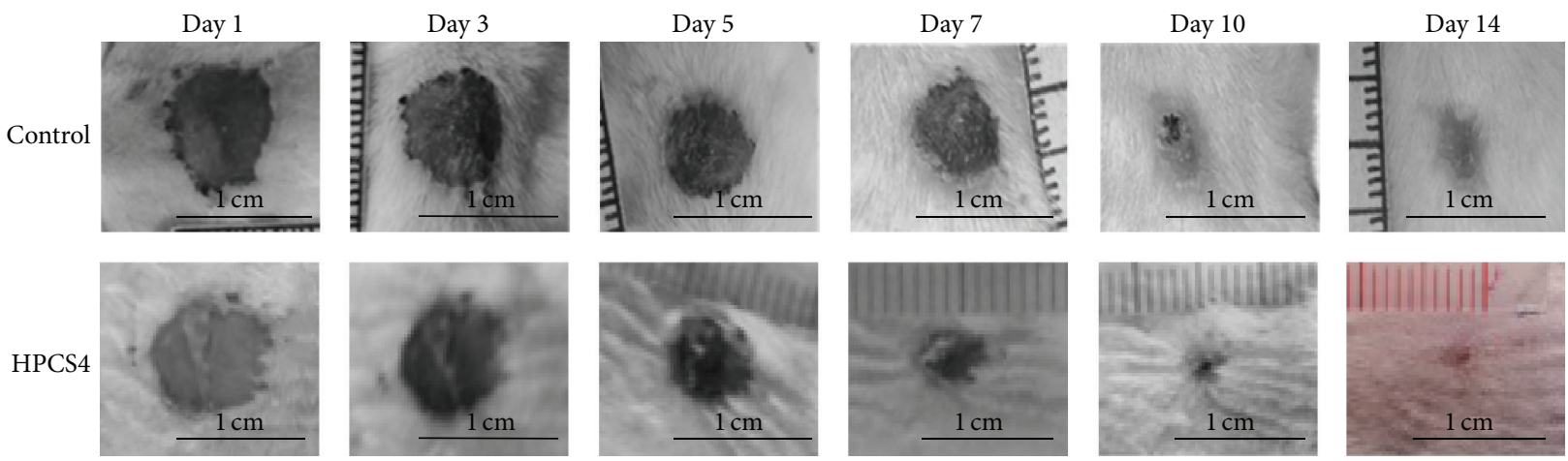

(a)

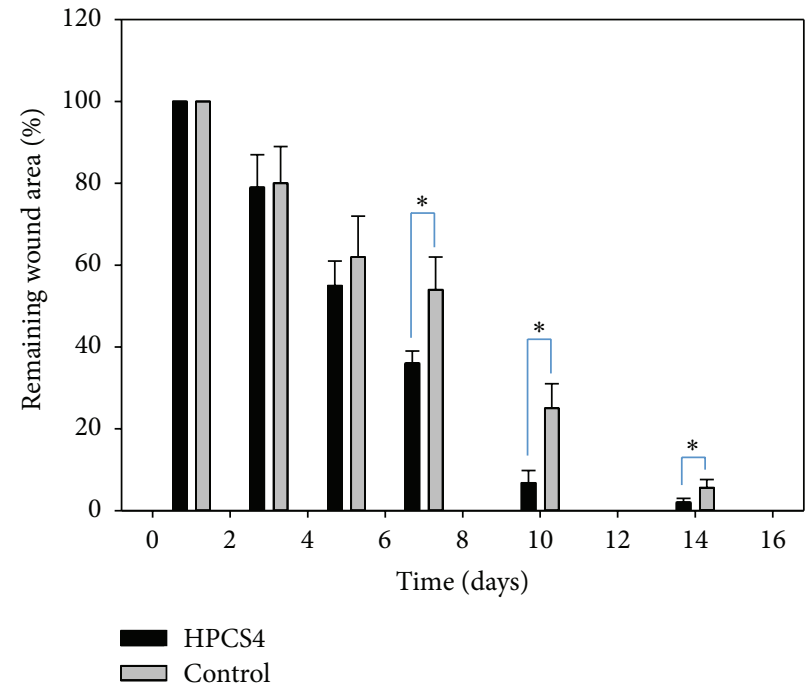

(b)

FIGURE 7: Photographs of treated wound compared with untreated wound after implantation (a) and percentage of remaining wound areas implanted with sample HPCS4 and control at days $1,3,5,7,10$, and 14 after implantation (b), ${ }^{*} p<0.01$.

intends to be used as a bioadhesive for skin wound healing. The hydrogels were examined for their reaction effectiveness, structure, degradation time, antibacterial property, and biocompatibility to prove whether the hydrogel could fulfill the requirements or not.

In the first step of optimizing the reaction effectiveness, the effect of concentration of chitosan on the gelation process was studied first and used as the fixed condition to alter other factors. The results proved that increasing chitosan concentration led to the decrease of gelation time. For the purpose of decreasing the gelation time which will lead to the decrease in surgical time, which is very important when performing surgery on patients having more than one wound [3], the higher the concentration of chitosan is, the more preferred it is. Though chitosan concentration can be prepared to more than $2 \% \mathrm{w} / \mathrm{v}$, its instability to dissolve completely makes it hard to prepare. Hence, $2 \% \mathrm{w} / \mathrm{v}$ was chosen as the initial condition. Based on the initial amount 


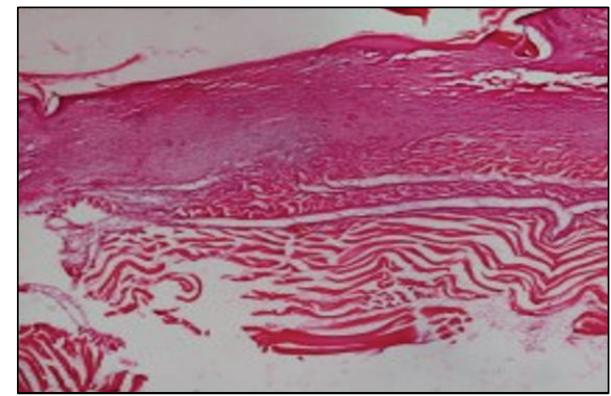

(a)

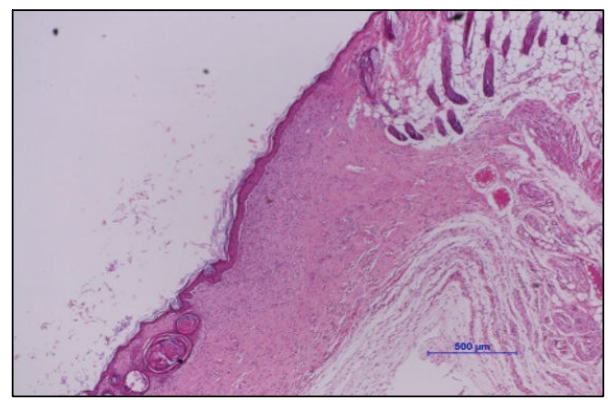

(c)

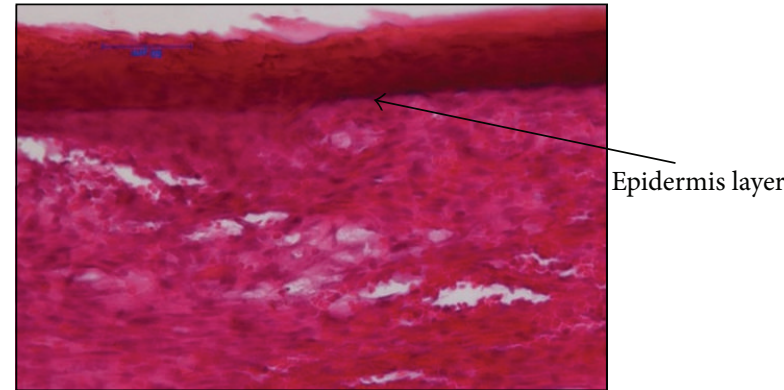

(b)

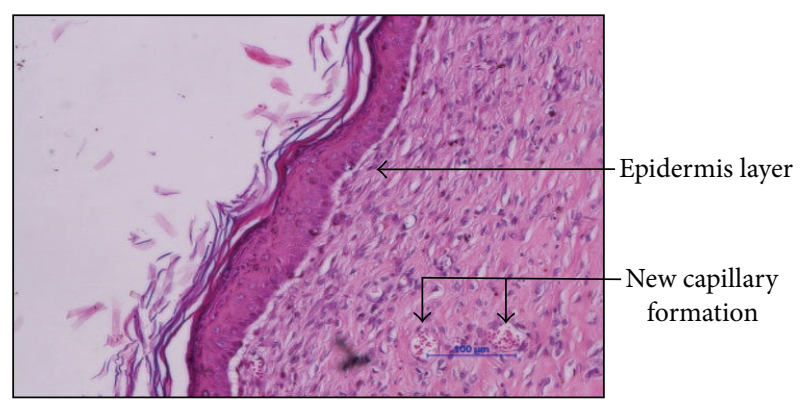

(d)

Figure 8: Optical images of H\&E staining of postimplantation at day 14 after implantation of the control mice at 4x (a) and 20x (b) magnifications and the HPCS4 implanted mice at $4 \mathrm{x}(\mathrm{c})$ and $20 \mathrm{x}(\mathrm{d})$ magnifications.

and concentration of chitosan, the effects of varying the amounts of PVPA and hyaluronan were studied and HPCS4 was lastly chosen as the optimum based on its reaction efficiency, which was represented by the formation of fibers, and viscoelasticity. With good viscoelasticity, HPCS4 could adhere to the skin edge when skin stretches or compresses. The final concentrations of hyaluronan, PVPA, and chitosan in the optimized hydrogel HPCS4 were $0.1 \%, 0.4 \%$, and $1.7 \%$, respectively.

The result from microstructure studies confirmed the success of fabricating new hydrogels by formation of electrostatic bonding between $\mathrm{NH}_{3}{ }^{+}$and acid groups [22, 23]. The hydrogel is an electrolyte complex that has a $3 \mathrm{D}$ porous structure with pore size ranging from 27 to $127 \mu \mathrm{m}$, which was suggested to reduce the wound contraction and enhance the proliferation of the preseeded cells [30].

Besides the porous structure, the wound healing process also requires a suitable degradation time of the hydrogel, with a degradation time of about two weeks, which is long enough for the duration of inflammation and proliferation stages in the wound healing process $[31,32]$. During these processes, the hydrogel would have served a multifunctional role, including hemostasis, prevention of external contamination, and framework for fibroblast cells seeding and building extracellular matrix (ECM) framework. After helping fibroblast cells to form the ECM framework for other cells' migration, proliferation, and differentiation, the hydrogel would have completed its roles, hence no longer needed. For that reason, two weeks would be a suitable degradation time for the hydrogel to be used as a skin adhesive.

Besides degradation rate, antibacterial activity is also an important factor. With its mild antibacterial activity against
E. coli, the hydrogel was suggested to have ability to prevent wound infections. Normally, decontamination process takes place in inflammatory stage. Yet if contamination cannot be cleaned effectively, it would prolong the stage, which may lead to the worst consequence of chronic wounds, which fail to be healed [33]. Hence, with this ability, the hydrogel could minimize the delay in wound healing process.

Biocompatibility of the hydrogel is another main issue that determines its applications in tissue engineering. In this study, the biocompatibility was evaluated in mice. The postimplantation zone showed no sign of inflammation during 14 days after treatment, which suggested that the hydrogel could generate no immune response and the wound was not infected or contaminated. Besides, the wound sizes of mice treated with hydrogel were reduced faster as compared to the untreated mice, suggesting that the hydrogel could have the ability to enhance wound healing process. Moreover, since the hydrogel covered the wound, it reduced the wound contraction, which is caused by myofibroblast cells to reduce the wound size, and hence minimized scar formation. After 14 days, the wound size reduced up to $98 \%$. The extracted results showed a complete structure of epidermal layer developed from adjacent keratinocytes [31], which means the wounds were sealed. In the inner layer, there are no signs of hydrogel residue, which means it was degraded completely, and the blood capillaries were being formed. In conclusion, the wound size after 14 days was mostly healed with no sign of fester and small size of scar remaining and the internal structure was forming. These results proved that HA/PVPA/CS hydrogel is a material that could enhance the wound healing process. 


\section{Conclusion}

In this research, a new type of hydrogel, HA/PVPA/CS hydrogel, was fabricated and its characteristics were examined. The hydrogel was optimized with the final concentration of $0.1 \%$, $0.4 \%$, and $1.7 \%$, of hyaluronan, PVPA, and chitosan, respectively. With highly porous structure, short gelation time, fast degradation rate, and ability to prevent $E$. coli infection and enhance wound healing process, HPCS4 hydrogel has fulfilled the basic requirements and has a potential in further studies to be used as a bioadhesive for skin wound healing application.

\section{Competing Interests}

The authors declare that they have no competing interests.

\section{Acknowledgments}

This research is funded by Vietnam National University-Ho Chi Minh City under Grant no. B2013-76-03.

\section{References}

[1] S. Khanlari and M. A. Dubé, "Bioadhesives: a review," Macromolecular Reaction Engineering, vol. 7, no. 11, pp. 573-587, 2013.

[2] M. L. B. Palacio and B. Bhushan, "Bioadhesion: a review of concepts and applications," Philosophical Transactions of the Royal Society A: Mathematical, Physical and Engineering Sciences, vol. 370, no. 1967, pp. 2321-2347, 2012.

[3] A. Soni, R. Narula, A. Kumar, M. Parmar, M. Sahore, and M. Chandel, "Comparing cyanoacrylate tissue adhesive and conventional subcuticular skin sutures for maxillofacial incisionsa prospective randomized trial considering closure time, wound morbidity, and cosmetic outcome," Journal of Oral and Maxillofacial Surgery, vol. 71, no. 12, pp. 2152.e1-2152.e8, 2013.

[4] T. B. Bruns, H. K. Simon, D. J. McLario, K. M. Sullivan, R. J. Wood, and K. J. S. Anand, "Laceration repair using a tissue adhesive in a Children's Emergency Department," Pediatrics, vol. 98, no. 4, pp. 673-675, 1996.

[5] J. Quinn, A. Drzewiecki, M. Li et al., "A randomized, controlled trial comparing a tissue adhesive with suturing in the repair of pediatric facial lacerations," Annals of Emergency Medicine, vol. 22, no. 7, pp. 1130-1135, 1993.

[6] P. J. M. Bouten, M. Zonjee, J. Bender et al., "The chemistry of tissue adhesive materials," Progress in Polymer Science, vol. 39, no. 7, pp. 1375-1405, 2014.

[7] M. N. V. R. Kumar, "A review of chitin and chitosan applications," Reactive \& Functional Polymers, vol. 46, no. 1, pp. 1-27, 2000.

[8] R. Jayakumar, M. Prabaharan, P. T. Sudheesh Kumar, S. V. Nair, and H. Tamura, "Biomaterials based on chitin and chitosan in wound dressing applications," Biotechnology Advances, vol. 29, no. 3, pp. 322-337, 2011.

[9] A. Niekraszewicz, "Chitosan medical dressings," Fibres \& Textiles in Eastern Europe, vol. 13, no. 6, pp. 16-18, 2005.

[10] W. Paul and C. P. Sharma, "Chitosan and alginate wound dressings-a short review," Trends in Biomaterials and Artificial Organs, vol. 18, no. 1, pp. 18-23, 2004.
[11] J. S. Frenkel, "The role of hyaluronan in wound healing," International Wound Journal, vol. 11, no. 2, pp. 159-163, 2014.

[12] F. Gao, Y. Liu, Y. He et al., "Hyaluronan oligosaccharides promote excisional wound healing through enhanced angiogenesis," Matrix Biology, vol. 29, no. 2, pp. 107-116, 2010.

[13] D. C. West, I. N. Hampson, F. Arnold, and S. Kumar, "Angiogenesis induced by degradation products of hyaluronic acid," Science, vol. 228, no. 4705, pp. 1324-1336, 1985.

[14] T.-W. Chung and Y.-L. Chang, "Silk fibroin/chitosan-hyaluronic acid versus silk fibroin scaffolds for tissue engineering: promoting cell proliferations in vitro," Journal of Materials Science: Materials in Medicine, vol. 21, no. 4, pp. 1343-1351, 2010.

[15] J. A. Burdick and G. D. Prestwich, "Hyaluronic acid hydrogels for biomedical applications," Advanced Materials, vol. 23, no. 12, pp. H41-H56, 2011.

[16] R. A. Franco, A. Sadiasa, and B.-T. Lee, "Utilization of PVPA and its effect on the material properties and biocompatibility of PVA electrospun membrane," Polymers for Advanced Technologies, vol. 25, no. 1, pp. 55-65, 2014.

[17] T. Kusunoki, M. Oshiro, F. Hamasaki, and T. Kobayashi, "Polyvinylphosphonic acid copolymer hydrogels prepared with amide and ester type crosslinkers," Journal of Applied Polymer Science, vol. 119, no. 5, pp. 3072-3079, 2011.

[18] J. Tan, R. A. Gemeinhart, M. Ma, and W. Mark Saltzman, "Improved cell adhesion and proliferation on synthetic phosphonic acid-containing hydrogels," Biomaterials, vol. 26, no. 17, pp. 3663-3671, 2005.

[19] L. MacArie and G. Ilia, "Poly(vinylphosphonic acid) and its derivatives," Progress in Polymer Science, vol. 35, no. 8, pp. 10781092,2010

[20] M. Fan, Y. Ma, J. Mao, Z. Zhang, and H. Tan, "Cytocompatible in situ forming chitosan/hyaluronan hydrogels via a metal-free click chemistry for soft tissue engineering," Acta Biomaterialia, vol. 20, pp. 60-68, 2015.

[21] Q. Chang, H. Gao, S. Bu, W. Zhong, F. Lu, and M. Xing, "An injectable aldehyded 1-amino-3,3-diethoxy-propane hyaluronic acid-chitosan hydrogel as a carrier of adipose derived stem cells to enhance angiogenesis and promote skin regeneration," Journal of Materials Chemistry B, vol. 3, no. 22, pp. 4503-4513, 2015.

[22] F. Göktepe, S. Ü. Çelik, and A. Bozkurt, "Preparation and the proton conductivity of chitosan/poly(vinyl phosphonic acid) complex polymer electrolytes," Journal of Non-Crystalline Solids, vol. 354, no. 30, pp. 3637-3642, 2008.

[23] Z. Ghasemi, R. Dinarvand, F. Mottaghitalab, M. EsfandyariManesh, E. Sayari, and F. Atyabi, "Aptamer decorated hyaluronan/chitosan nanoparticles for targeted delivery of 5-fluorouracil to MUC1 overexpressing adenocarcinomas," Carbohydrate Polymers, vol. 121, pp. 190-198, 2015.

[24] P. H. Weigel, V. C. Hascall, and M. Tammi, "Hyaluronan synthases," The Journal of Biological Chemistry, vol. 272, no. 22, pp. 13997-14000, 1997.

[25] N. Thi-Hiep, D. V. Hoa, and V. V. Toi, "Injectable in situ crosslinkable hyaluronan-polyvinyl phosphonic acid hydrogels for bone engineering," Journal of Biomedical Science and Engineering, vol. 6, no. 8, pp. 854-862, 2013.

[26] Z. Durmus, H. Erdemi, A. Aslan, M. S. Toprak, H. Sozeri, and A. Baykal, "Synthesis and characterization of poly(vinyl phosphonic acid) (PVPA)- $\mathrm{Fe}_{3} \mathrm{O}_{4}$ nanocomposite," Polyhedron, vol. 30, no. 2, pp. 419-426, 2011. 
[27] A. G. B. Pereira, E. C. Muniz, and Y.-L. Hsieh, " ${ }^{\text {11H NMR }}$ and ${ }^{1} \mathrm{H}-{ }^{13} \mathrm{C}$ HSQC surface characterization of chitosan-chitin sheath-core nanowhiskers," Carbohydrate Polymers, vol. 123, pp. 46-52, 2015.

[28] C. D. G. Abueva and B.-T. Lee, "Poly(vinylphosphonic acid) immobilized on chitosan: a glycosaminoglycan-inspired matrix for bone regeneration," International Journal of Biological Macromolecules, vol. 64, pp. 294-301, 2014.

[29] S. D. E. Nath, C. Abueva, B. Kim, and B. T. A. Lee, "Chitosanhyaluronic acid polyelectrolyte complex scaffold crosslinked with genipin for immobilization and controlled release of BMP2," Carbohydrate polymers, vol. 115, pp. 160-169, 2015.

[30] I. V. Yannas, E. Lee, D. P. Orgill, E. M. Skrabut, and G. F. Murphy, "Synthesis and characterization of a model extracellular matrix that induces partial regeneration of adult mammalian skin," Proceedings of the National Academy of Sciences of the United States of America, vol. 86, no. 3, pp. 933-937, 1989.

[31] M. Shankar, B. Ramesh, D. Roopa Kumar, and M. Niranjan Babu, "Wound healing and it's important-a review," Der Pharmacologia Sinica, vol. 1, no. 1, pp. 24-30, 2014.

[32] P. Chandika, S.-C. Ko, and W.-K. Jung, "Marine-derived biological macromolecule-based biomaterials for wound healing and skin tissue regeneration," International Journal of Biological Macromolecules, vol. 77, pp. 24-35, 2015.

[33] S. Guo and L. A. DiPietro, "Critical review in oral biology \& medicine: factors affecting wound healing," Journal of Dental Research, vol. 89, no. 3, pp. 219-229, 2010. 

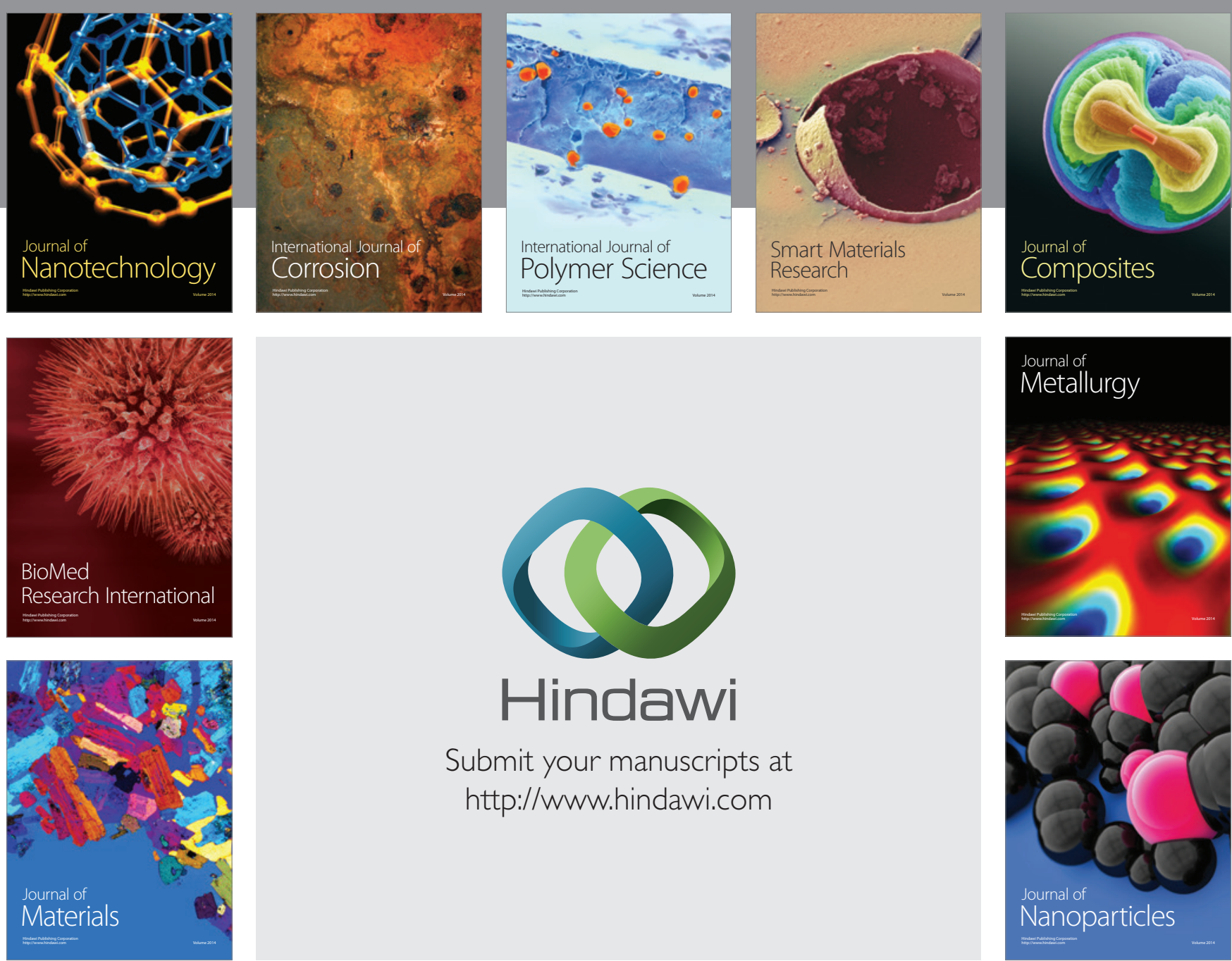

\section{Hindawi}

Submit your manuscripts at

http://www.hindawi.com

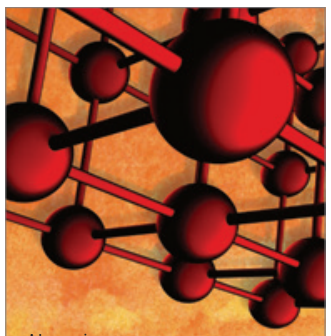

Materials Science and Engineering
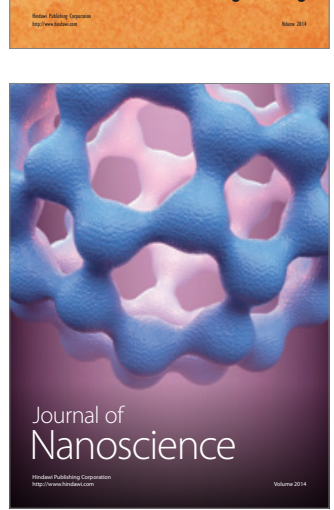
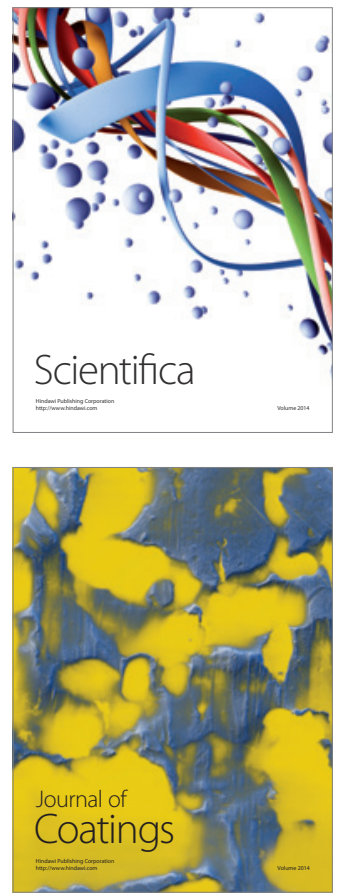
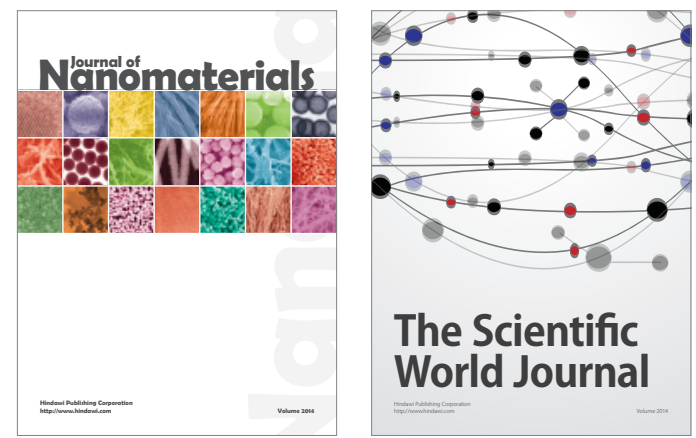

The Scientific World Journal
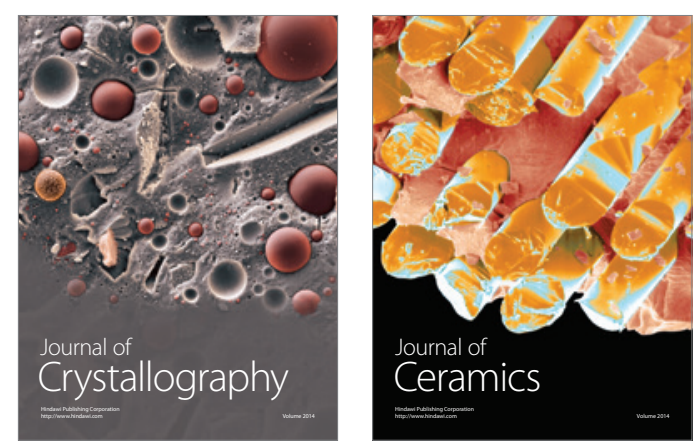
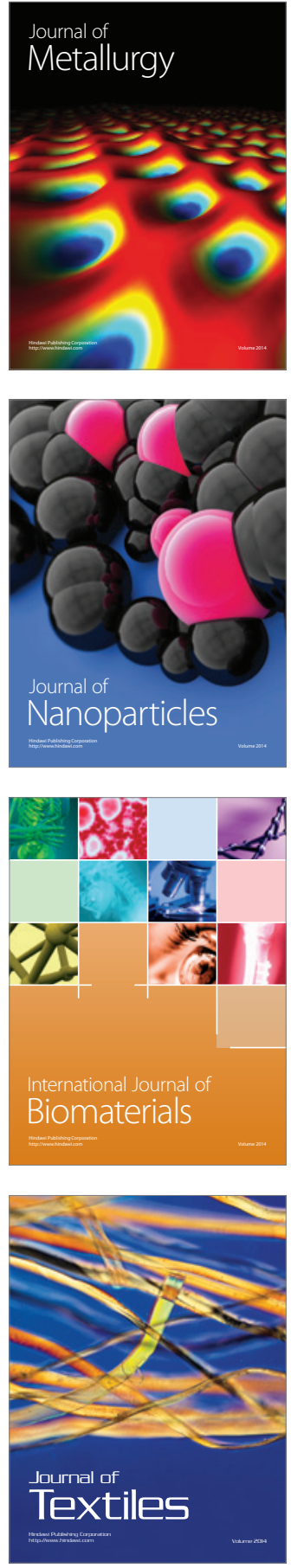